\title{
Ansiedade de separação em adultos \\ com transtorno de pânico: um tratamento cognitivo-comportamental ${ }^{1}$
}

\section{Separation anxiety in adults with panic disorder: a cognitive-behavior treatment}

\author{
Ana Gabriela Pinheiro da Silva ANNICCHINO² \\ Evandro Gomes de MATOS 3
}

\begin{abstract}
Resumo
Este estudo foi desenvolvido com pacientes adultos, ambulatoriais, com transtorno de pânico segundo os critérios do Manual de Estatísticas e Diagnósticos-IV. O objetivo foi avaliar a incidência e a severidade de sintomas de ansiedade de separação e o efeito da terapia cognitivo-comportamental sobre eles. O processo psicoterapêutico baseou-se no manual "Vencendo o Pânico". Foram também utilizados Inventário Beck de Depressão, Escala Sheehan de Ansiedade, e o Mood Anxiety Spectrum Project. Os resultados demonstraram a minimização ou o desaparecimento dos sintomas de ansiedade de separação após o tratamento, bem como o decréscimo nos graus de ansiedade e depressão. Pode-se afirmar, portanto, que há sintomas da ansiedade de separação no adulto, e que a terapia cognitivo-comportamental apresenta-se como eficaz tanto quanto no tratamento do transtorno de pânico, provavelmente, por serem os mesmos sistemas que fundamentam os pensamentos em ambos os casos.
\end{abstract}

Unitermos: adulto; ansiedade da separação; terapia cognitivo-comportamental; transtorno de pânico.

\begin{abstract}
This study was developed with adult patients, all of them panic disorder clinically diagnosed according to the Manual de Estatisticas e Diagnósticos-IV criteria. The aim of the study was to investigate the incidence and severity of Separation Anxiety symptoms among these patients and the effect of Cognitive-Behavior Therapy on these symptoms. The psychotherapy process was based on the Panic treatment manual, "Overcoming Panic." Also used were the Beck Depression Inventory, Sheehan Anxiety Scale and the Mood Anxiety Spectrum Project. The results showed reduction or disappearance of Separation Anxiety symptoms after the treatment, as well as decrease in the degrees of anxiety and depression. It can be affirmed therefore, that there is Separation Anxiety in the adult, and that Cognitive-Behavior Therapy is efficient in the treatment of both Separation Anxiety and Panic Disorder, probably, because the same systems are at the base of the thoughts for both.
\end{abstract}

Uniterms: adult; separation anxiety; cognitive behavior therapy; panic disorder.

\section{$\boldsymbol{\nabla \nabla \nabla \nabla}$}

1 Artigo elaborado a partir da dissertação de A.G.P.S. ANNICCHINO, intitulada "Incidência e severidade da ansiedade de separação antes e após o tratamento cognitivo-comportamental, em pacientes com transtorno de pânico". Departamento de Psicologia Médica e Psiquiatria, Faculdade de Ciências Médicas, Universidade Estadual de Campinas, 2006.

2 Consultório Particular. R. Bento Dias, 888, Pão de Açúcar, 16360-000, Capivari, SP, Brasil. Correspondência para/Correspondence to: A.G.P. S. ANNICCHINO. E-mail: <anagabrielaann@uol.com.br>.

3 Universidade Estadual de Campinas, Faculdade de Ciências Médicas, Departamento de Psicologia Médica e Psiquiatria. Campinas, SP, Brasil. 
Este trabalho teve como objetivo investigar a incidência de sintomas de ansiedade de separação (AS) em pacientes adultos com transtorno de pânico (TP), segundo o item A do domínio 1 do Mood Anxiety Spectrum Project (SCI-PAS) (Cassano et al.,1997) e submetê-los a um programa específico de tratamento cognitivo-comportamental, verificando se há redução e/ou remissão dos sintomas de AS.

Em 1980, o TP e o transtorno de ansiedade generalizada (TAG) surgiram como divisão da neurose de angústia no Manual de Estatísticas e Diagnósticos III - DSM III (APA, 1980). Os ataques de pânico (AP) assumiram importância como queixa e o seu papel na patogênese dos transtornos ansiosos, especialmente da agorafobia (AGO), foi ressaltado. O manual dividiu também a AGO em com e sem AP (Andrade et al., 1997).

Mais tarde, o DSM IV (APA, 1995) trouxe novas mudanças: considerou que AP e a esquiva fóbica podem apresentar-se como sintomas em outros quadros clínicos além do TP e da AGO e ampliou o diagnóstico de TAG para a infância. Referiu ainda a existência de AP limitados, com presença de menos de quatro sintomas.

A AS foi descrita no DSM-IV como um transtorno da infância, no qual a separação de uma criança de sua figura de ligação, geralmente a mãe, é acompanhada de ansiedade excessiva. Deve ter duração mínima de quatro semanas e a presença de pelo menos três dos seguintes sintomas: sofrimento excessivo e recorrente diante da ocorrência ou previsão de afastamento de casa ou de figuras importantes de vinculação; preocupação persistente e excessiva a cerca de perder ou sobre possíveis perigos envolvendo figuras importantes de vinculação; de que um evento indesejado leve à separação de uma figura importante de vinculação; relutância ou recusa em ir para a escola ou a qualquer outro lugar por medo da separação; relutância em dormir sem estar próximo a uma figura importante de vinculação ou a pernoitar longe de casa; temor excessivo e persistente ou relutância em ficar sozinho ou sem as figuras importantes de vinculação em casa ou sem adultos significativos em outros contextos; pesadelos envolvendo o tema da separação; repetidas queixas de sintomas somáticos (tais como cefaléias, dores abdominais, náuseas ou vômitos) quando a separação de figuras importantes de vinculação ocorre ou é prevista. A perturbação deve iniciar-se antes dos dezoito anos; causar sofrimento significativo, ou prejuízo no funcionamento social, acadêmico ou em outras áreas importantes da vida do indivíduo. Além disso, não deve ocorrer durante o curso de um transtorno invasivo do desenvolvimento, esquizofrenia ou outro transtorno psicótico e, em adolescentes e adultos, não é mais bem explicada por TP com AGO.

Souza e Matos (1996) descreveram que o transtorno de AS pode manifestar-se de várias maneiras, desde a dificuldade em dormir sozinho até a recusa em ir ou permanecer na escola.

Gutiérrez (1996) caracterizou como AP a reação apresentada por crianças com AS ao separarem-se de seus pais. A relação entre TP e AS vem sendo objeto de estudo de muitos autores, com diferentes resultados, entretanto muitos apontam para a existência de tal associação. Klein e Gittelman (1984) observaram a presença de taxas mais elevadas de AS e fobia escolar em pacientes com TP. Deltito, Perugi, Maremmani, Mignani e Cassano (1986) referiram que, em um estudo, 60\% dos pacientes com TP e AGO apresentaram AS na infância. Kagan, Reznick, Snidman, Gibbons e Johnson (1988) observaram que no decorrer da vida o comportamento inibido assume expressões diferenciadas em cada faixa etária. Primeiro como AS na infância ou evitação social, mais tarde - na adolescência - como fobia social e TP na fase adulta. Souza (1996) descreveu que, de 68 pacientes com TP, 32\% preenchiam critérios para o diagnóstico de AS na infância ou adolescência.

Embora fosse a AS um transtorno predominantemente da infância, Cassano et al. (1997), ao descreverem o espectro do pânico agorafóbico, mencionaram a presença de sintomas desse transtorno na vida adulta (Cyranowski et al., 2002). Definiram o espectro do pânico agorafóbico pela presença de sintomas atípicos, isolados ou parciais, que podem preceder ou acompanhar uma condição ainda não totalmente expressa. Para a sua avaliação são acessados sete domínios: sintomas de pânico; expectativa ansiosa; fobias e evitações; sensibilidade ao reasseguramento; sensibilidade a substâncias; sensibilidade ao estresse; sensibilidade à separação.

Examinando a relação entre o espectro do pânico agorafóbico e os transtornos mentais da infância e da adolescência, supôs-se que os sintomas desses influenciam o comportamento adulto e, mais tarde, são vistos como atípicos (Pini, Maser, Dell'Osso \& Cassano, 1998). 
Segundo Cassano et al. (1997) a AS em adultos pode manifestar-se como dificuldade em sair de casa a trabalho ou a passeio, dormir sozinho ou longe de casa e por reações dramáticas a perdas ou antecipação delas. Esses indivíduos tendem a desenvolver relacionamentos caracterizados pela dependência.

A terapia cognitivo-comportamental (TCC) tem obtido sucesso no tratamento dos transtornos ansiosos. Caracteriza-se por ser breve e por ter objetivos claros a serem alcançados. É prática e tanto o terapeuta quanto o paciente têm papéis ativos nas tarefas propostas (Otto \& Whittal, 1995). Pode ser utilizada sozinha ou em associação à medicação (Ham, Waters \& Oliver, 2005).

Pesquisas demonstraram que a TCC é bem tolerada, apresenta boa relação custo-efetividade e produz importantes benefícios para indivíduos com TP a curto e longo prazo (Landon \& Barlow, 2004).

Na TCC, a interpretação (pensamentos automáticos) feita pelo sujeito, diante de um estímulo ambiental, produz o sentimento (emoção) e por conseqüência o comportamento (Beck, 1998). Os sentimentos gerados evidenciam crenças e suposições que estruturam os pensamentos, tornando possível a descoberta das distorções cognitivas, específicas, que regem as doenças.

Nardi et al. (1996) ressaltaram que a terapia cognitiva, desenvolvida por Beck (1998), reconhece a ansiedade como resposta a uma ameaça, relacionando a ansiedade patológica aos processos de informação relativa ao perigo. A ansiedade é mantida pela falta de controle sobre o meio ambiente, dessa forma, pacientes ansiosos consideram-se incapazes e com poucos recursos para enfrentar ameaças.

No caso da ansiedade, o medo decorrente de uma situação na qual não há perigo denuncia distorções no processo cognitivo e indica a necessidade de intervenção (Rangé, 2001a). O tratamento consiste em eliminar padrões de funcionamento desadaptado, ou seja, o medo das sensações físicas, as evitações e as "estratégias de segurança" desenvolvidas pelo indivíduo (Otto \& Whittal, 1995).

\section{Método}

Neste estudo utilizou-se o método quase experimental com medidas antes e depois.

A amostra contou com 22 sujeitos de ambos os sexos, na faixa etária entre 24 e 52 anos (idade média de
37 anos), portadores de TP segundo os critérios do DSM IV. Desses, 16 concluíram o tratamento. Os pacientes provinham do Núcleo de Atendimento dos Transtornos de Ansiedade (NATA) no ambulatório geral de psiquiatria adulto do Hospital das Clínicas da Universidade de Campinas (Unicamp).

O projeto foi submetido ao Comitê de Ética em Pesquisa da Faculdade de Ciências Médicas da Unicamp e aprovado sem restrições com o parecer de no 038/2002. Os pacientes foram informados sobre os objetivos do estudo, assegurados quanto ao sigilo e convidados a assinar o Termo de Consentimento Livree Esclarecido.

Os critérios de inclusão foram: atender os critérios diagnósticos para TP, segundo o DSM IV; assinar o termo de consentimento; ser maior de 18 anos; preencher o protocolo NATA; estar em vigência de AP; responder a todos os instrumentos de pesquisa; e ter a presença de sintomas de AS segundo o domínio I da Structured Clinical Interview for Panic-Agoraphobic Spectrum (SCI-PAS).

Foram excluídos deste estudo: pacientes com distúrbios psicóticos; uso ou abuso de substâncias psicoativas ou transtornos mentais orgânicos; e os que não aceitaram preencher o termo de consentimento.

Empregaram-se instrumentos de avaliação como o Inventário Beck de Depressão (BDI) - seguindo os escores: 0 a 9 - sem depressão ou mínima; 10 a 18 - depressão leve; 19 a 29 - depressão moderada e acima de 30 - depressão grave. A Escala de Sheehan de Ansiedade (Sheehan) avaliou subjetivamente a intensidade dos sintomas das ansiedades endógena (apenas essa foi utilizada neste trabalho) e exógena (Caetano, 1986), respeitando os escores referentes à ansiedade endógena: 0 a 30 - leve; 31 a 50 - moderada; 51 a 80 - marcada; e acima de 81 - severa. No domínio I da SCI-PAS (versão em português adaptada por Matos, Sardelli, Ravera, D’Agostino \& Matos, 2005), o paciente responde sim ou não a dez questões, em dois momentos: alguma vez na vida (lifetime) e no último mês. Utilizou-se, também, o protocolo NATA para levantar dados de identificação, características sociodemográficas e idade do primeiro AP. 
O processo de TCC baseou-se no manual de tratamento denominado "Vencendo o Pânico: instruções passo a passo para quem trata ataques de pânico (VP)" (Rangé, 2001b).

Os pacientes foram submetidos a uma sessão de TCC, individual, por semana, totalizando 12 sessões. Foram utilizadas estratégias terapêuticas consideradas efetivas, tais como: informações sobre o problema e o método de tratamento, treino de relaxamento, reestruturação cognitiva, exposição interoceptiva, reestruturação de crenças perfeccionistas e de padrões de comportamento não assertivo.

Tarefas de casa foram solicitadas e envolveram a prática dos exercícios trabalhados em sessão, leitura de textos relacionados ao assunto e a realização de atividades propostas.

A seguir, são detalhados os objetivos de cada sessão, segundo Rangé (2001b).

- 1a Sessão - pré-teste - objetivos: avaliação geral do problema; informação sobre a pesquisa; preenchimento do Termo de Consentimento; preenchimento da Ficha de Caracterização de Casos.

- $2^{a}$ e $3 a$ Sessões - pré-teste - objetivos: aplicação de questionários que fazem parte do manual, mas que, contudo, não foram utilizados nesta pesquisa. Na $3^{a}$ sessão foi realizada também a aplicação da SCI-PAS.

- 4a Sessão - intervenção - objetivos: rapport, informação ao paciente sobre o TP e o tratamento oferecido, coleta de informações e estabelecimento de metas para o tratamento, leitura dos textos: "Transtorno de Pânico"; "Fisiologia da Hiperventilação" e "Fisiologia e Psicologia do Medo e da Ansiedade".

- 5 a Sessão - intervenção - objetivos: treinamento de habilidades de manejo - aplicação da estratégia A.C.A.L.M.E.-S.E. e a introdução das técnicas comportamentais de exposição interoceptiva e de controle de respiração e iniciação do registro de pensamentos disfuncionais (RPD), que foi feito durante todas as semanas até o final do tratamento, tendo sido feitas, em cada sessão, as correções.

- 6aSessão - intervenção-objetivos: conscientização corporal - discussão dos textos; análise de RPD e proposta de respostas alternativas, relaxamento físico;

36 exposição interoceptiva.
- 7aSessão-intervenção-o bjetivos: fortalecimento da auto-eficácia - análise de RPD; discussão, com o paciente, da primeira crença irracional de Ellis (1962): "É absolutamente necessário por mim ser amado e aprovado pelas pessoas que me são importantes". Início do treino assertivo que tem como primeiro passo fazer e receber elogios. Para pacientes com AGO, foi solicitada a construção da hierarquia de ansiedade para exposição situacional.

8 a Sessão - intervenção - objetivos: manejo existencial - checagem do RPD; segundo passo do treino de assertividade: dizer não a exigências consideradas não razoáveis; início do treino de exposição situacional à primeira situação da hierarquia criada pelo paciente; discussão da segunda crença de Ellis (1962):"Para se ter valor, é necessário ser competente e bem-sucedido em todos os aspectos da vida" e curtograma.

- ga Sessão - intervenção - objetivos: manejo existencial (continuação) - análise do RPD e do curtograma, novas exposições a situações da hierarquia de ansiedade; terceiro passo do treino de assertividade - a solicitação de coisas ou ajuda de outras pessoas; discussão da terceira crença de Ellis (1962): "É terrível e catastrófico quando as coisas não acontecem do jeito que eu queria". Execução da lista de desejos.

- 10a Sessão - intervenção - objetivos: manejo existencial (continuação) - análise do RPD e da realização de atividades mencionadas como prazerosas no curtograma; incentivo ao planejamento de um futuro feliz e realizador baseado na lista de desejos; novas exposições situacionais; quarto passo do treino de assertividade que encoraja a solicitação de mudanças de comportamento a outras pessoas; discussão de situações negativas da vida e a idéia de pânico como "freio", baseando-se no texto de mesmo nome.

- 11aSessão-intervenção-objetivos:encerramento (manutenção e prevenção de recaída) - revisão das tarefas e repetição dos exercícios de relaxamento e exposição interoceptiva; informação sobre a prevenção de recaídas e avaliação do trabalho pelo paciente.

- 12a Sessão - pós-teste - objetivos: avaliação do status do paciente em relação aos níveis de ansiedade, depressão, funcionamento global na vida e a alteração produzida na AS - reaplicação dos instrumentos: Sheehan; BDI e SCI-PAS. 


\section{Resultados}

Dos 22 pacientes iniciais, $18(81,82 \%)$ eram mulheres e $4(18,18 \%)$ homens. A maioria (40,91\%) tinha apenas o ensino fundamental, seguido pelo nível superior $(31,82 \%)$ e pelo ensino médio $(27,27 \%)$, respectivamente. Cinqüenta e nove por cento tinham um (a) companheiro (a), enquanto $41,00 \%$ estavam sozinhos.

A intensidade da sintomatologia depressiva, ansiosa e de AS, antes e depois da intervenção, está registrada na Tabela 1.

Para a correlação entre os graus de depressão e ansiedade com os de AS (alguma vez na vida e último mês), aplicou-se a correlação de Spearman (Tabela 2).

Pode-se comprovar a existência de uma relação entre os graus de depressão (BDI) e ansiedade (Sheehan) com a AS no último mês, com os respectivos $r=0,55$ e $r=0,7168$, consideradas relações média e alta. Isso indica que o tratamento teve efeito positivo devido à existência da relação do decréscimo dos graus de ansiedade e depressão conjuntamente com os graus de AS.

Para fazer a relação entre a freqüência de sintomas de AS antes e depois do tratamento, optou-se por criar categorias de acordo com o número de respostas afirmativas dadas pelo sujeito e que equivaleram à quantidade de sintomas apresentados, a fim de facilitar a análise. As categorias foram: 0 a 4; 5 a 7 e 8 a 10.

Em relação às freqüências, observou-se que, após a intervenção, houve uma queda dos graus de AS devido ao aumento do número de pessoas no grupo 0 a 4 para a variável último mês depois. Para alguma vez na vida, como os valores são os mesmos, não houve alteração (Tabela 3).

Foi aplicado o teste de Wilcoxon pareado para verificar se após a intervenção houve queda significativa dos graus de AS de acordo com o último mês. Para alguma vez na vida, não foi aplicado, pois as colunas são iguais (Tabela 4).

\section{Discussão}

De acordo com o DSM IV, o grau de tolerância à separação aceitável pode ser determinado culturalmente, pois há culturas em que é valorizada a forte interdependência entre os membros da família.
Tabela 1. Médias (M) e desvios-padrão (DP) dos sintomas de depressão e ansiedade antes e após a intervenção.

\begin{tabular}{|c|c|c|c|}
\hline Variáveis & $\mathrm{n}$ & M & DP \\
\hline Depressão (BDI) antes & 16 & $\begin{array}{c}2,94 \\
\text { (moderada) }\end{array}$ & 0,85 \\
\hline Depressão (BDI) depois & 16 & $\begin{array}{c}2,13 \\
\text { (leve) }\end{array}$ & 1,15 \\
\hline Ansiedade(Sheehan) antes & 16 & $\begin{array}{c}3,19 \\
\text { (marcada) }\end{array}$ & 0,75 \\
\hline Ansiedade(Sheehan) depois & 16 & $\begin{array}{c}2,06 \\
\text { (moderada) }\end{array}$ & 1,24 \\
\hline Alguma vez na vida antes (SCI-PAS) & 21 & 8,43 & 1,36 \\
\hline Último mês antes (SCI-PAS) & 16 & 6,81 & 2,43 \\
\hline Alguma vez na vida depois (SCI-PAS) & 21 & 8,43 & 1,36 \\
\hline Último mês depois (SCI-PAS) & 16 & 3,31 & 2,91 \\
\hline
\end{tabular}

Tabela 2. Estimativas de correlação entre sintomas de depressão e ansiedade com a ansiedade de separação (alguma vez na vida e último mês).

\begin{tabular}{|c|c|c|}
\hline \multirow{2}{*}{ Variáveis } & \multicolumn{2}{|c|}{ Correlações } \\
\hline & Antes & Depois \\
\hline \multirow[t]{2}{*}{ Depressão vs alguma vez na vida } & 0,114 & 0,095 \\
\hline & $(p$-valor $=0,6743)$ & $(p$-valor $=0,7258)$ \\
\hline \multirow[t]{2}{*}{ Depressão vs último mês } & 0,2459 & $0,55^{*}$ \\
\hline & $(p$-valor $=0,3585)$ & $(p$-valor $=0,0273)$ \\
\hline \multirow[t]{2}{*}{ Ansiedade vs alguma vez na vida } & $-0,1894$ & 0,2623 \\
\hline & $(p$-valor $=0,4823)$ & $(p$-valor $=0,3264)$ \\
\hline \multirow[t]{2}{*}{ Ansiedade vs último mês } & $-0,1546$ & $0,7168^{*}$ \\
\hline & $(p$-valor $=0,5674)$ & $(p$-valor $=0,0018)$ \\
\hline
\end{tabular}

Tabela 3. Distribuição dos escores de ansiedade de separação antes e depois da intervenção.

\begin{tabular}{lcc}
\hline Categorias & $n$ & $\%$ \\
\hline Alguma vez na vida (antes e depois) & 4 & 19,05 \\
5 a 7 & 17 & 80,95 \\
8 a 10 & 21 & 100,00 \\
\hline Total & & \\
\hline Último mês (antes) & 2 & 12,50 \\
0 a 4 & 7 & 43,75 \\
5 a 7 & 7 & 73,75 \\
8 a 10 & 16 & 100,00 \\
\hline Total & & \\
\hline Último mês (depois) & 11 & 68,75 \\
0 a 4 & 3 & 18,75 \\
5 a 7 & 2 & 12,50 \\
8a 10 & 16 & 100,00 \\
\hline Total & & \\
\hline
\end{tabular}

Tabela 4. Comparação entre último mês antes e último mês depois.

\begin{tabular}{lccccccc}
\hline Variáveis & $\mathrm{n}$ & $\mathrm{M}$ & $\mathrm{DP}$ & Mediana & Mín. & Máx. & $p$ \\
\hline Último mês antes & 16 & 6,81 & 2,43 & 7,0 & 3 & 10 & \\
Último mês depois & 16 & 3,31 & 2,91 & 2,5 & 0 & 9 & $0,0026^{*}$ \\
\hline
\end{tabular}

M: média; DP: desvio-padrão; Min.: mínimo; Max.: máximo. 
A AS é mais freqüente em mulheres e acomete 4\% das crianças e adolescentes jovens. Pode haver maior freqüência do transtorno em filhos de mães que apresentam TP e também em parentes biológicos de primeiro grau.

De acordo com o DSM IV, o TP sem AGO é duas vezes mais freqüente e, nas mulheres, o TP com AGO aparece com freqüência três vezes maior. A prevalência do TP durante a vida chega a 3,5\%. Há um predomínio de seu surgimento entre o final da adolescência e os 30 anos. Parentes em primeiro grau de indivíduos portadores de TP apresentam uma chance de quatro a sete vezes maior de desenvolver o transtorno.

Deltito et al. (1986), em um grupo de pacientes com TP e AGO, observaram que 60\% manifestaram AS na infância. Contudo essa associação não se aplicou ao grupo de pacientes com TP sem AGO.

Raskin, Peeke, Dickman e Pinsker (1982) encontraram, em 17 pacientes adultos com TP, 35\% de AS.

Os resultados obtidos neste trabalho estão de acordo com estudos como o de Cassano et al. (1997), que descreveu a presença de sintomas de AS no adulto.

Manicavasagar, Silove e Curtis (1997) relataram que um terço de 44 pacientes que participaram de seus estudos referiram o início dos primeiros sintomas de AS já na vida adulta. Os autores sugeriram que há necessidade de examinar mais sistematicamente a forma como a AS pode ocorrer na vida adulta.

Cyranowsky et al. (2002) desenvolveram a Strucutured Clinical Interview for Separation Anxiety Symptoms (SCI-SAS) e destacaram a praticabilidade e a utilidade clínica de acessar os sintomas da AS, experimentados durante a vida adulta, com um instrumento apropriado à idade.

Manicavasagar, Silove, Wagner e Drobny (2003) também apontaram para a possibilidade de diagnóstico de AS na vida adulta. Os adultos podem demonstrar intensa ansiedade ao serem separados de esposas, filhos ou parentes. Essa experiência traz imposições à vida da pessoa, pela necessidade de proximidade com suas figuras de afeiçoamento.

Esses dados são consistentes ao que apresentou o DSM IV ao referir o transtorno em jovens - embora 38 esse manual não reconheça AS na vida adulta. Nos indivíduos mais velhos o transtorno pode limitar as suas capacidades em lidar com situações novas (mudança de emprego, casamento), além de criar uma preocupação excessiva em relação aos filhos ou cônjuge e manifestar intenso desconforto ao separar-se deles.

A resposta do transtorno de AS no adulto ao tratamento, baseado na TCC, reproduz a eficácia dessa abordagem constatada por Dia (2001). A TCC como coadjuvante no tratamento de transtornos ansiosos tem sido cada vez mais recomendada por apresentar resultados efetivos tanto para adultos como para crianças.

Apesar de demonstrar resultados satisfatórios aos seus objetivos, a pesquisa contou com uma amostra pequena, o que impossibilitou outras correlações, uma vez que estatisticamente não apresentaram significância. A realização de um estudo mais amplo, com um número maior de sujeitos e a utilização de um instrumento mais completo e específico para acessar a AS, como, por exemplo, a Adult Separation Anxiety Semistructured Interview (ASA-SI) (Manicavasagar et al., 2003) ou a SCI-SAS, forneceria novos dados e resultados mais seguros.

\section{Conclusão}

Estão presentes, no adulto, sintomas de AS, indicando que esse transtorno pode estender-se além da infância com características clínicas diferenciadas pela maturidade, e que há, portanto, necessidade de investigá-lo melhor nessa população.

A diminuição da sintomatologia de AS produziu também redução das sintomatologias ansiosa e depressiva (embora não significativa, talvez por ser uma amostra pequena).

A TCC e, mais especificamente, o VP, demonstraram-se eficazes tanto no tratamento do TP quanto da AS. Essa abordagem pode apresentar-se como uma interessante alternativa a ser utilizada tanto no serviço público quanto no privado.

\section{Referências}

American Psychiatric Association (APA). (1980). Diagnostic and Statistical Manual of Mental Disorders (DSM-III). 3rd ed. Washington, DC: American Psychiatric Association. 
Andrade, L. H. S. G., Lotufo-Neto, F., Gentil, V., Maciel, L. M. A., Shavitt, R. G., \& Bernick, M. A. (1997). Classificação e diagnóstico dos transtornos ansiosos. In V. Gentil, F. Lotufo-Neto \& M. A. Bernick. Pânico, fobias e obsessões: a experiência do projeto AMBAN (3a.ed., pp.37-53). São Paulo: Universidade de São Paulo.

Associação Psiquiátrica Americana (APA). (1995). Manual estatístico e diagnóstico de transtornos mentais (DSM IV). 4a. ed. Porto Alegre: Artes Médicas.

Beck, A. T., Rush, A. J., Shaw, B. F., \& Emery, G. (1998). Terapia cognitiva da depressão. Porto Alegre: Artes Médicas.

Caetano, D. (1986). Comparação entre pacientes com desordem de pânico e prolapso da valva mitral e aqueles com desordem de pânico e sem prolapso de valva mitral. Jornal Brasileiro de Psiquiatria, 35 (1), 53-59.

Cassano, G. B., Michelini, S., Shear, M. K., Coli, E., Maser, J. D., \& Frank, E. (1997). The panic-agoraphobic spectrum: a descriptive approach to the assessment and treatment of subtle symptoms. American Journal of Psychiatry, 154 (6), 27-38.

Cyranowski, J. M., Shear, M. K., Rucci, P., Fagiolini, A., Frank, E., \& Grochocnski, V. J. (2002). Adult separation anxiety: psychometric properties of a new structured clinical interview. Journal Psychiatric Research, 36 (2), 77-86.

Deltito, J. A., Perugi, G., Maremanni, I., Mignani, V., \& Cassano, G. B. (1986). The importance of separation anxiety in the differentiation of panic disorder from agoraphobia. Psychiatric Developments, 4 (3), 227-236.

Dia, D. A. (2001). Cognitive-behavioral therapy with a sixyear-old boy with separation anxiety disorder: a case study. Health \& Social Work, 26 (2), 125-128.

Ellis, A. (1962). Reason and emotion in psycotherapy. New York: Lyle Stuart.

Gutierrez, A. J. L. (1996). Ansiedade de separação y transtorno de pânico. Psiquiatria Biológica, 4 (2), 109-112.

Ham, P., Waters, D. B., \& Oliver, M. N. (2005). Treatment of panic disorder. American Family Physician, 71 (4), 733-739.

Kagan, J., Reznick, J. S., Snidman, N., Gibbons, J., \& Johnson, M. O. (1988). Childhood derivatives of inhibition and lack of inhibition to the unfamiliar. Child Development, 59 (6), 1580-1589.

Klein, D. F., \& Gittelman, R. (1984). Relationship between separation anxiety and panic and agoraphobic disorders. Psychopathology, 17 (Suppl 1), 56-65.

Landon, T. M., \& Barlow, D. H. (2004). Cognitive-behavioral treatment for panic disorder: current status. Journal Psychiatric Practice, 10 (4), 211-226.

Manicavasagar, V., Silove, D., \& Curtis, J. (1997). Separation anxiety in adulthood: a phenomenological investigation. Comprehensive psychiatry, 38 (5), 274-282.
Manicavasagar, V., Silove, D., Wagner, R., \& Drobny, J. (2003). A self-report questionnaire for measuring separation anxiety in adulthood. Comprehensive Psychiatry, 44 (2), 146-1453.

Matos, E. G., Sardelli, L. R., Ravera, L., D'Aagostino, G., \& Matos, T. M. G. (2005). Versão para o português de um novo instrumento para o diagnóstico do espectro do transtorno do pânico (PAS-SR). Jornal Brasileiro de Psiquiatria, 54 (2), 108-113.

Nardi, A. E., Mendlowicz, M., Figueira, I., Andrade, Y., Camisao, C., Marques, C., et al. (1996). Transtorno de ansiedade generalizada - I: questões teóricas e diagnósticas. Jornal Brasileiro de Psiquiatria, 45 (3), 173-178.

Otto, M. W., \& Whittal, M. L. (1995). Cognitive-behaviour therapy and the longitudinal course of panic disorder. Psychiatric Clinics of North American, 18 (4), 803-820.

Pini S., Maser J. D., Dell'Osso L., \& Cassano G. B. (1998). Origins of the panic-agoraphobic spectrum and its implications for comorbidity. CNS Spectrums, 4 (3), 49-56.

Rangé, B. (2001a). Vencendo o pânico: instruções passo-a-passo para quem sofre de ataques de pânico. Rio de Janeiro: Instituto de Psicologia, Universidade federal do Rio de Janeiro.

Rangé, B. (2001 b). Vencendo o pânico: instruções passo-a- passo para quem trata de ataques de pânico. Rio de Janeiro: Instituto de Psicologia, Universidade Federal do Rio de Janeiro.

Raskin, M., Peeke, V. S., Dickman, W., \& Pinsker, H. (1982). Panic and generalized anxiety disorders: developmental antecedents and precipitants. Archives of General Psychiatry, 39 (6), 687-689.

SAS Institute Inc. (1999-2001). SAS/STAT softwarechangesand enhancements though release 8.2. Cary, NC: SAS Institute, Inc.

Snedecor, W. G., \& Cochram, W. G. (1989). Statistical Methods. 8th ed. lowa State.

Souza, A. M. (1996). Estudo da associação entre ansiedade de separação e transtorno de pânico. Dissertação de mestrado não-publicada, Departamento de Psicologia e Psiquiatria Médica, Faculdade de Ciências Médicas, Universidade Estadual de Campinas.

Souza, A. M., \& Matos, E. G. (1996). Fobia escolar em gêmeos univetelínicos - um preditor para fobia social? Jornal Brasileiro de Psiquiatria, 45 (12), 695-698.

Recebido em: 22/11/2005

Versão final reapresentada em: 21/8/2006

Aprovado em: 15/9/2006 
